\title{
Effect of a Various Additivies to the Formation of Aqueous Biphasic System Polyetylenglycole (Peg)/Sodium Citrate/Water
}

\author{
Gunel M. Shahbazova*, Eldar A. Masimov \\ Department of Physics, Baku State University, Baku, Azerbaijan \\ Email: ^shahbazova.gunel@mail.ru, masimovspektr@rambler.ru
}

How to cite this paper: Shahbazova, G.M. and Masimov, E.A. (2022) Effect of a Various Additivies to the Formation of Aqueous Biphasic System Polyetylenglycole (Peg)/Sodium Citrate/Water. Open Access Library Journal, 9: e8142.

https://doi.org/10.4236/oalib.1108142

Received: November 1, 2021

Accepted: January 17, 2022

Published: January 20, 2022

Copyright $\odot 2022$ by author(s) and Open Access Library Inc.

This work is licensed under the Creative Commons Attribution International License (CC BY 4.0).

http://creativecommons.org/licenses/by/4.0/

\begin{abstract}
Liquid-liquid equilibrium data for aqueous biphasic system formed by polyethylene glycol (PEG)/sodium citrate $\left(\mathrm{C}_{6} \mathrm{H}_{5} \mathrm{O}_{7} \mathrm{Na}_{3}\right) /$ water $\left(\mathrm{H}_{2} \mathrm{O}\right)$ were determined at $298.15 \mathrm{~K}$, respectively. Binodal data were correlated using a fourparameter equation. The effect of the molecular weight of PEG $(1500,3000$, 6000 ) on the binodal curves was investigated, and it was found that the two-phase region expands with an increase in the molecular weight of PEG. The effects of some monohydric (methanol, ethanol, propanol) and polyhydric alcohols (ethylene glycol, glycerin) on the formation of biphasic system PEG/sodium citrate were examined simultaneously. It was found that the phase diagram binodal in the presence of ethylene glycol and glycerin to the system, obtained that binodal curve is slipped to the top of coordinate at the high concentrations, two-phase systems occur with a higher concentration of components which formed phases. This means that ethylene glycol and glycerin acts as a factor that destroys the structure of water, breaking the hydrogen bonds between water molecules.
\end{abstract}

\section{Subject Areas}

Biophysics

\section{Keywords}

Polyetylenglycole, Sodium Citrate, Alcohols, Ethylene Glycol, Glycerin

\section{Introduction}

Aqueous two-phase systems (ATPS) are aqueous solutions of a combination of two incompatible polymers or a polymer and salt that separate into two phases 
when their concentration surpasses a certain threshold value [1] [2] [3] [4] [5]. One phase is rich in one polymer, and the second phase is rich in the other polymer as (or salt) with water as a solvent in both phases. These phases differ in almost all physico-chemical properties, including their relative hydrophobicity. This difference is reflected in the value of the distribution coefficient of the substances entering the system of the phases. Water as the main component of both phases in ATPS forms a gentle environment for biomolecules to separate and polymers stabilize their structure and biological activities [4] [5] [6] [7]. Phase separation in ATPS is affected by different factors like, concentration and molecular weight (MW) of polymer, concentration and composition of salt [8] [9].

But two-polymer and polymer-salt (e.g., phosphate, sulfate or citrate) systems have grown rapidly and a lot of work has been put into studying this technique using these types of ATPSs [10] [11]. This method has advantages over conventional extraction techniques like, environment-friendly, low cost, capable of continuous operation, ease of scaling-up and is efficient for many kinds of experiments specially for the concentration and purification of biomolecules [10] [12] [13] [14].

The main purpose of this work setting the phase diagrams of PEG/sodium citrate/ water biphasic systems and the explaining of mechanism formation of phases by analyzing them. To achieve this goal, were put forward the following issues:

- Setting the binodal curves of the PEG/sodium citrate/water two-phase systems and determining the equation of connecting line.

- Study of the effect of the molecular weight of the polymer and alcohols on the phase diagram (binodal curve) of the PEG/sodium citrate/water two-phase system.

The study of these effects can contribute to the formation of two-phase systems and to the understanding of the mechanisms of distribution of substances in these systems and to the improvement of their application.

\section{Experimental Section}

\subsection{Materials}

In this work using PEG with $M_{n}=6000(3000,4000)$ molecular weight which produced by "Panreac" firm, of Spain and "chemical cleaner" and "special cleaner" salts, sodium citrate, alcohols. All experiments were conducted with doubledistillation water in quartz plates.

PEGs of different molecular weights are widely used polymers in ATPS due to their low toxicity, low price, and low volatile nature [8]. Polyethylene glycol has a low toxicity and is used in a variety of products [15]. The polymer is used as a lubricating coating for various surfaces in aqueous and non-aqueous environments [16]. In biophysics, polyethylene glycols are the molecules of choice for the functioning ion channels diameter studies, because in aqueous solutions they have a spherical shape and can block ion channel conductance [17] [18]. 
Sodium citrate is the sodium salt of citric acid. It is white, crystalline powder or white, granular crystals, slightly deliquescent in moist air, freely soluble in water, practically insoluble in alcohol. Like citric acid, it has a sour taste. From the medical point of view, it is used as alkalinizing agent. It works by neutralizing excess acid in the blood and urine. It has been indicated for the treatment of metabolic acidosis [19].

\subsection{Methods}

To prepare the initial solution of PEG (usually $40 \%$ - 50\%), a certain amount of polymer was weighed on the scales and dissolved in water for 10 - 15 minutes by heating in a water bath at $100^{\circ} \mathrm{C}$. In order to sterilize, the polymer solution was filtered while hot and stored in $20 \mathrm{~g}$ containers in the refrigerator. Aqueous solutions of salts of different molar concentrations were prepared and then expressed as a percentage.

Two-phase polymer-inorganic electrolyte systems were obtained by mixing the initial solutions of polymer and salt. Usually, 2 grams systems were prepared. To prepare the system of the given composition, the initial solutions were weighed on a test tube and up to 2 grams of water were added. After that, system was stirred intensively until a thermodynamic equilibrium was reached. To speed up the process, the mixed systems were centrifuged for 20 minutes at a frequency of $4000 \mathrm{rpm}$ (revolutions per minute). Different experiments have shown that the thermodynamic equilibrium conditions obtained by centrifuging the systems or waiting 6 hours are the same.

One of the main characteristics of two-phase aqueous polymer systems is their binodal curve. The binodal curve is the geometric location of the points in the phase diagram that separate the single-phase (homogeneous) region of the system from the two-phase (heterogeneous) region. Two methods are usually used to construct the binodal curve, the turbidity method and the methods for studying the phase composition of the system.

With the first method, $1 \mathrm{~g}$ of each of the initial solutions prepared to construct the binodal curve is mixed. If the point obtained belongs to a two-phase region, then a visible turbid solution (because it is a system that is not in thermodynamic equilibrium) is obtained. By adding water to this system, we note the critical point that passes from the two-phase region to the single-phase region. By increasing the number of these points, we obtain critical points in the phase diagram that move from a two-phase region to a single-phase region, where the geometric location is the binodal curve of the system. With this method, the volumes of the simultaneously existing phases of the system were determined and the volume ratio was used to determine the angle of inclination of the connecting line.

The binodal curves constructed by the second method, is the method of determining the phase composition of the system, become more accurate, and when we construct this curve with this method, we also obtain a connecting line. For 
this purpose, an initial system is prepared according to the point falling in the two-phase region. Thermodynamic equilibrium is achieved by waiting for six hours or centrifuging. The phases of the system are separated, and the composition of these phases is determined separately. Amount of PEG in phases determined with Centro photometric method, amount of salt is determined with refractometric method. Thus, points indicating the composition of the upper and lower phases are obtained for the system corresponding to the initial point taken. The line passing through these three points is called the connecting line. By taking the point corresponding to the initial system near and far from the origin, we obtain the binodal curve of the system and the connecting line.

The experiments were performed at room temperature $\left(20^{\circ} \mathrm{C}-25^{\circ} \mathrm{C}\right)$.

\section{Results and Discussions}

In this work physico-chemical properties of PEG- $\mathrm{C}_{6} \mathrm{O}_{7} \mathrm{H}_{5} \mathrm{Na}_{3}-\mathrm{H}_{2} \mathrm{O}$ two-phase systems were studied. For this purpose, polyethylene glycol (PEG)-6000, sodium salt of citric acid (chemically pure) and bidistilled water were used. The binodal curve of this system was constructed, and the angle of inclination of the connecting line was determined. It should be noted that compared to polymerpolymer-water and polymer-inorganic electrolyte two-phase systems, the process of phasing in this system is faster, within 1 hour.

One of the main characteristics of polymer-water two-phase systems is its binodal curve. Figure 1 shows the binodal curve, the connecting line (CL) of a two-phase system given by an aqueous solution of citric acid and polyethylene glycol with a molecular weight of $\mathrm{Mn} \sim 6000$, as well as the equation found by the least squares method of CL

As can be seen from the equations, the tangent of the angle of inclination of

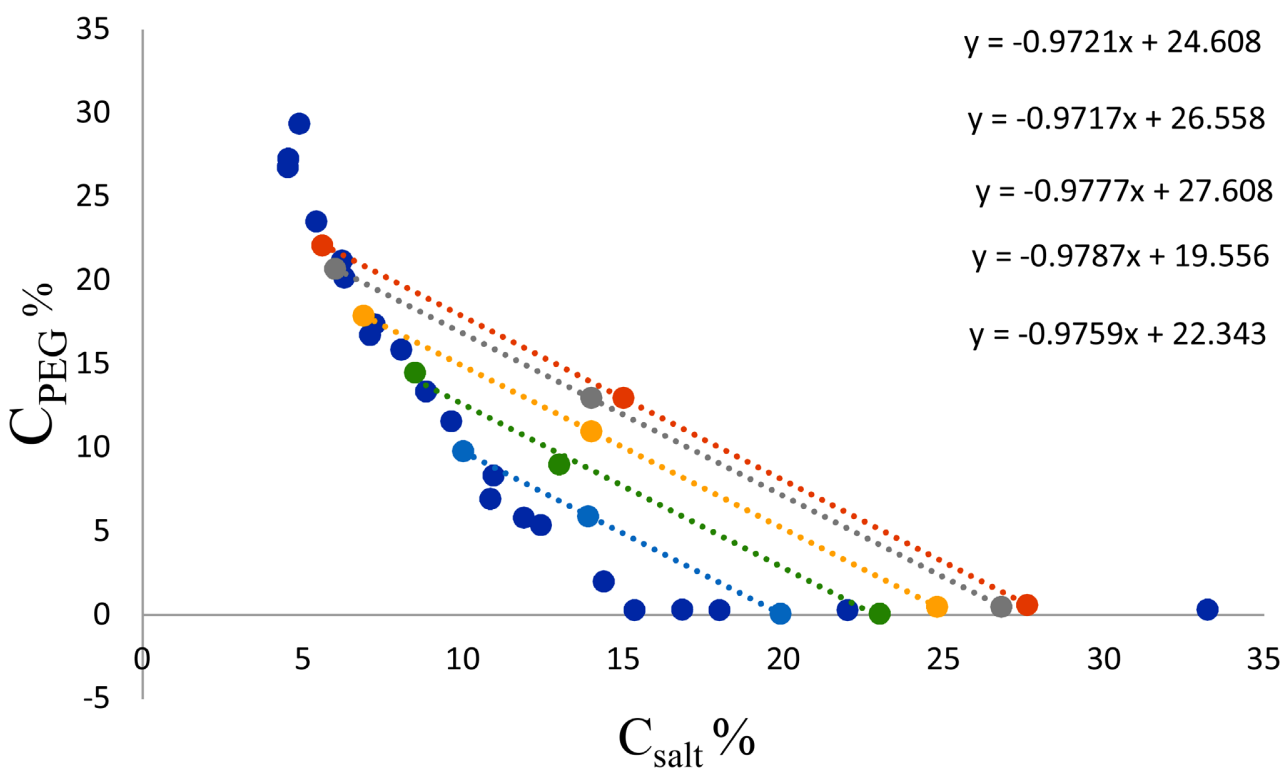

Figure 1. Binodal curve and connecting line of aqueous biphasic system PEG $(\mathrm{Mn}=6000)$ /sodium citrate/water. 
CL is $\operatorname{tg} \alpha=0.97$. Note that for this system the angle of inclination of CL is constant.

Generally, binodal of two-phase aqueous polymer systems is understand that the geometrical place of transition points from one-phase oblast to two-phase oblast in coordinate system which consist of concentration of polymers. In the figure, the region below the binodal curve is called a single-phase or homogeneous region, and the region above is called a two-phase or heterogeneous region. In other words, according to the coordinates of any point taken from the upper region, the system will be divided into two phases when it becomes thermodynamic equilibrium. One of the phases of the system is enriched with any one of components. The asymmetry of the binodal curve is known to be due to the difference in the molecular masses of the components of the system.

In Table 1 given polymer and salt compositions of the systems corresponding to different points taken from the heterogeneous region of the biphasic system PEG $\left(M_{n}=6000\right) /$ sodium citrate and the simultaneously existing phases of these primary systems.

The process of phase separation is associated with the formation of two different water structures as a result of the different interactions of water with its components. In the studied PEG- $\mathrm{C}_{6} \mathrm{O}_{7} \mathrm{H}_{5} \mathrm{Na}_{3}-\mathrm{H}_{2} \mathrm{O}$ biphasic system, phase separation also occurs due to the formation of water of two different structures. It is clear that under the influence of any external factor affecting the structure of the water will be accompanied by a change in the main characteristics of the state diagram of the system. One such external effect may be the molecular weight of the polymer, which is one of the phase-forming components. Figure 2 shows the effect of the molecular weight of polyethylene glycol on the phase diagram of the biphasic system PEG (1500, 3000, 6000)/sodium citrate/water.

As can be seen from Figure 2, as the molecular weight of PEQ increases, the process of phasing at smaller concentrations of the components takes place. This fact can be explained by the fact that as the molecular weight of the polymer increases, the number of water molecules in its hydrate layer increases and the number of free water molecules in the system decreases, dissolution becomes more difficult, and different structural water phases occur. That is, the system is

Table 1. Liquid-liquid equilibrium data for PEG 6000 + sodium citrate + water systems at 298.15 .

\begin{tabular}{cccccccc}
\hline \multirow{2}{*}{ Tie line } & \multicolumn{2}{c}{ Overall } & \multicolumn{2}{c}{ top phase } & \multicolumn{2}{c}{ Bottom phase } & \multirow{2}{*}{$\operatorname{tg} \alpha_{B X}$} \\
\cline { 2 - 6 } & PEG \% & Salt \% & PEG \% & Salt \% & PEG \% & Salt\% & \\
\hline 1 & 20.3 & 13.9 & 0.6 & 21.8 & 40.4 & 5.8 & -0.967 \\
2 & 14 & 14 & 0.8 & 18.2 & 38.2 & 6 & -0.975 \\
3 & 14 & 15 & 0.6 & 20.4 & 35.4 & 6.2 & -0.9721 \\
4 & 16 & 14 & 0.6 & 19.2 & 37.2 & 6 & -0.9717 \\
5 & 18 & 12 & 1 & 17.8 & 36 & 6.2 & -0.9777 \\
\hline
\end{tabular}




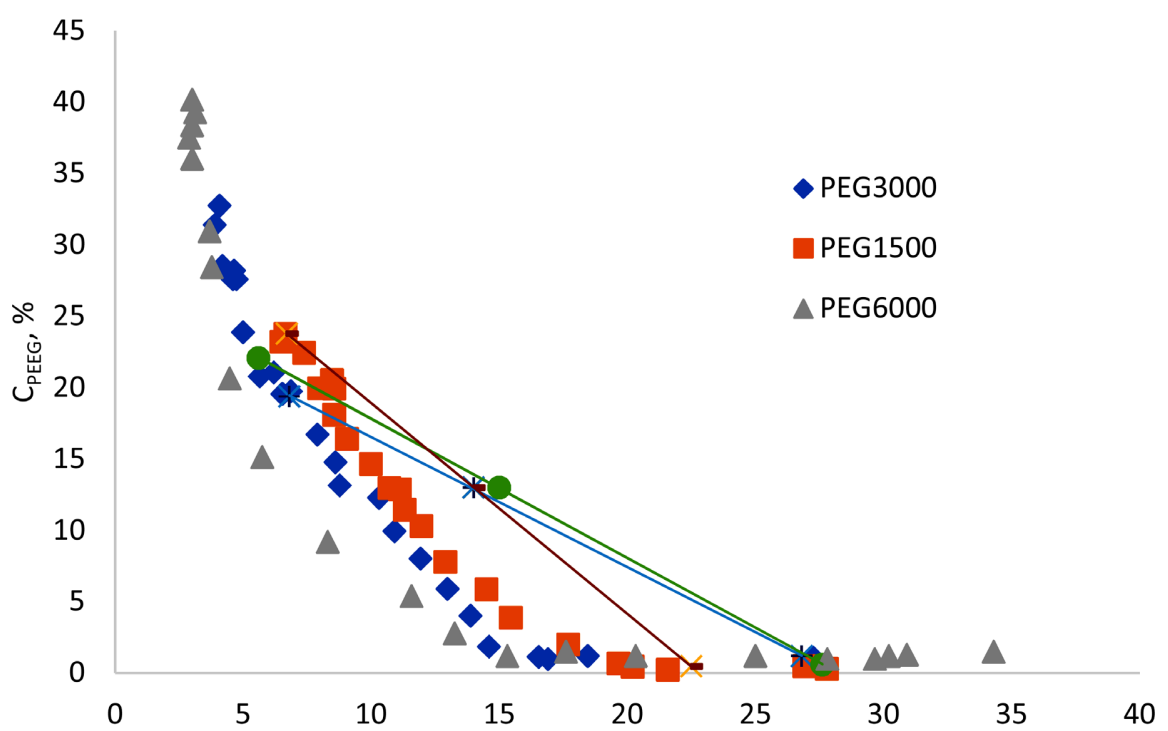

Figure 2. Phase diagram of the biphasic system PEG (1500, 3000, 6000)/sodium citrate.

Table 2. Changes of critical point with change in molecular weight of PEG.

\begin{tabular}{lccc}
\hline & $\mathrm{C}_{\text {cr }}$ PEG & $\mathrm{C}_{\text {cr }}$ salt & tg $\alpha$ \\
\hline PEG (1500)- $\mathrm{C}_{6} \mathrm{H}_{5} \mathrm{O}_{7} \mathrm{Na}_{3}-\mathrm{H}_{2} \mathrm{O}$ & 7.4 & 12.7 & 1.47 \\
PEG (3000)- $\mathrm{C}_{6} \mathrm{H}_{5} \mathrm{O}_{7} \mathrm{Na}_{3}-\mathrm{H}_{2} \mathrm{O}$ & 1.87 & 14.6 & 0.91 \\
PEG (6000)- $\mathrm{C}_{6} \mathrm{H}_{5} \mathrm{O}_{7} \mathrm{Na}_{3}-\mathrm{H}_{2} \mathrm{O}$ & 0.5 & 15.35 & 0.975 \\
\hline
\end{tabular}

divided into phases more easily.

Table 2 shows the concentrations of the components at the critical point of the angle of tendency and binodal, which is one of the main characteristics of the system under the influence of molecular weight.

The effects of some monohydric [20] and polyhydric alcohols on the PEG$\mathrm{C}_{6} \mathrm{H}_{5} \mathrm{O}_{7} \mathrm{Na}_{3}-\mathrm{H}_{2} \mathrm{O}$ system were examined simultaneously (Figure 3 ).

The results show that, with the influence of ethylene glycol, glycerin to the system, obtained that binodal curve is slipped to the top of coordinate at the high concentrations, two-phase systems occurred with a higher concentration of components which formed phases. This means that ethylene glycol and glycerin acts as a factor that destroys the structure of water, breaking the hydrogen bonds between water molecules. As a result, the number of free water molecules increases, the phase-forming components are easier to dissolve, and the phase separation occurs at higher concentrations of the components.

It should be noted that propanol was the most influential among the alcohols in the process of phase separation (Figure 4). At the same time, the effect of different concentrations of ethanol $(0.5 \mathrm{~mol} / \mathrm{l})$ and propanol $(0.3,0.5 \mathrm{~mol} / \mathrm{l})$ on the binodal curve of the system was studied. It has been found that as the concentration of propanol increases, the phase separation occurs at lower concentrations of the phase-forming components. Thus, a systematic study of the effect of alcohols on the binodal curves of PEG- $\mathrm{C}_{6} \mathrm{H}_{5} \mathrm{O}_{7} \mathrm{Na}_{3}-\mathrm{H}_{2} \mathrm{O}$ systems 


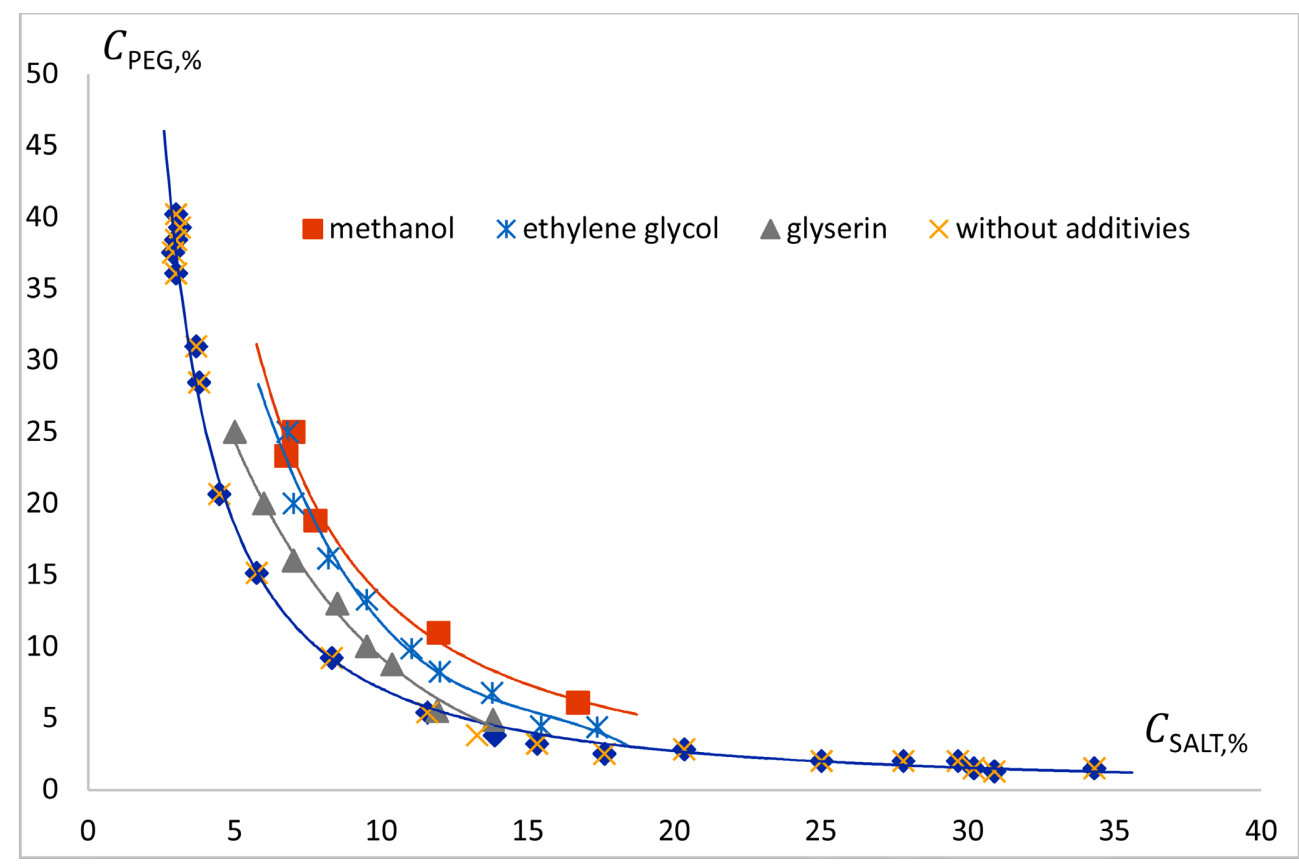

Figure 3. Effect of polyhydric alcohols on the binodal curve of the two-phase system PEG/sodium citrate.

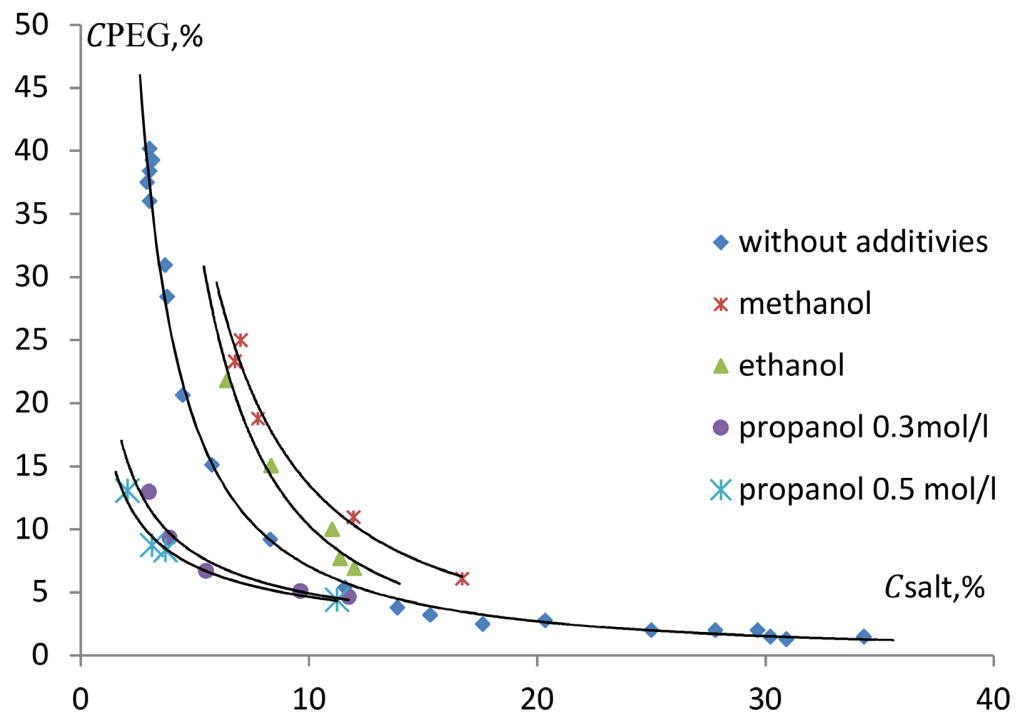

Figure 4. Effect of monohydric alcohols on the binodal curve of the two-phase system PEG/sodium citrate

from external factors revealed that ethanol and propanol have a structural effect on the structure of water and accelerate the phase separation process.

\section{Conclusions}

The formation of a two-phase system is observed in an aqueous mixture of polyethylene glycol with sodium citrate at thermodynamic equilibrium. A phase diagram of the acquired two-phase system (binodal curve and tendency angle of connecting or tie line) is investigated. 
- The effect of the molecular weight of PEG $(1500,3000,6000)$ on the binodal curves was investigated, it was found that the two-phase region expands with an increase in the molecular weight of PEG.

- The effects of some monohydric (methanol $\left(\mathrm{CH}_{3} \mathrm{OH}\right)$, ethanol $\left(\mathrm{CH}_{3} \mathrm{CH}_{2} \mathrm{OH}\right)$, (propanol $\left(\mathrm{CH}_{3} \mathrm{CH}_{2} \mathrm{CH}_{2} \mathrm{OH}\right)$ and polyhydric alcohols (ethylene glycol, glyserin) on the formation of biphasic system PEG/sodium citrate were examined. It was found that that ethanol and propanol have a structural effect on the structure of water and accelerate the phase separation process [20]. The phase diagram (binodal) in the presence of methanol $\left(\mathrm{CH}_{3} \mathrm{OH}\right)$ and ethanol $\left(\mathrm{CH}_{3} \mathrm{CH}_{2} \mathrm{OH}\right)$ is mixed upwards to increase the homogeneous region of the diagram, the smaller the displacement, the greater the number of hydrophobic functional groups in the alcohol molecule [20].

- With the influence of ethylene glycol, glycerin to the system, obtained that binodal curve is slipped to the top of coordinate at the high concentrations, two-phase systems occur with a higher concentration of components which formed phases. This means that ethylene glycol and glycerin acts as a factor that destroys the structure of water, breaking the hydrogen bonds between water molecules. As a result, the number of free water molecules increases, the phase-forming components are easier to dissolve, and the phase separation occurs at higher concentrations of the components.

So that, conclusion these above, we can come to a conclusion that, any highmolecular or low-molecular matters include water, are change the available structure and corresponding state.

\section{Conflicts of Interest}

The authors declare no conflicts of interest.

\section{References}

[1] Zaslavsky, B.Y., Gulaeva, N.D., Djafarov, S.F., Masimov, E.A. and Miheeva, L.M. (1990) Phase Separation in Aqueous Poly(ethylene glycol)- $\left(\mathrm{NH}_{4}\right)_{2} \mathrm{SO}_{4}$ Systems and Some Physicochemical Properties of the Phases. Journal of Colloid and Interface Science, 137, 147-156. https://doi.org/10.1016/0021-9797(90)90051-O

[2] Toledo, M.O., Farias, F.O., Igarashi-Mafra, L. and Mafra, M.R. (2019) Salt Effect on Ethanol-Based Aqueous Biphasic Systems Applied to Alkaloids Partition: An Experimental and Theoretical Approach, Journal of Chemical \& Engineering Data, 64, 2018-2026. https://doi.org/10.1021/acs.jced.8b01024

[3] Zaslavsky, B.Y., Bagirov, T.O., Borovskaya, A.A., et al. (1986) Aqueous Biphasic Systems Formed by Nonionic Polymers I. Effects of Inorganic Salts on Phase Separation. Colloid and Polymer Science, 264, 1066-1071.

https://doi.org/10.1007/BF01410324

[4] Carvalho, C.P., Coimbra, J.S.R. and Costa, I.A.F., Minim, L.A. and Maffia, M.C. (2008) Influence of the Temperature and Type of Salt on the Phase Equilibrium of peg 1500 + Potassium Phosphate and peg 1500 + Sodium Citrate Aqueous Two-Phase Systems. Química Nova, 31, 209-213. https://doi.org/10.1590/S0100-40422008000200004

[5] Albertsson, P.Å. (1986) Partition of Cell Particles and Macromolecules. Wiley In- 
tersciences, Hoboken.

[6] Ruiz-Ruiz, F., Benavides, J., Aguilar, O. and Rito-Palomares, M. (2012) Aqueous Two-Phase Affinity Partitioning Systems: Current Applications and Trends. Journal of Chromatography A, 1244, 1-13. https://doi.org/10.1016/j.chroma.2012.04.077

[7] Hatti-Kaul, R. (2001) Aqueous Two-Phase Systems. Molecular Biotechnology, 19, 269-277. https://doi.org/10.1385/MB:19:3:269

[8] Espitia-Saloma, E., Vázquez-Villegas, P., Aguilar, O. and Rito-Palomares, M. (2014) Continuous Aqueous Two-Phase Systems Devices for the Recovery of Biological Products. Food and Bioproducts Processing, 92, 101-112. https://doi.org/10.1016/j.fbp.2013.05.006

[9] Zafarani-Moattar MT, Hamzehzadeh S, Nasiri S. (2012) A New Aqueous Biphasic System Containing Polypropylene Glycol and a Water-Miscible Ionic Liquid. Biotechnology Progress, 28, 146-156. https://doi.org/10.1002/btpr.718

[10] Glyk, A., Solle, D., Scheper, T. and Beutel, S. (2015) Optimization of PEG-Salt Aqueous Two-Phase Systems by Design of Experiments. Chemometrics and Intelligent Laboratory Systems, 149, 12-21. https://doi.org/10.1016/j.chemolab.2015.09.014

[11] Masimov, E., Abbasov, H., Bagirov, T., Hasanova, H. and Gurbanov, J.T. (2007) Structural Features of the Two-Phase System Polyethylene Glycol- $\mathrm{C}_{4} \mathrm{O}_{6} \mathrm{H}_{4} \mathrm{Na}_{2}-\mathrm{H}_{2} \mathrm{O}$. Journal Physics Institute of Physics of the National Academy of Sciences of Azerbaijan, 13, 334-336.

[12] Mazzola, P.G., Lopes, A.M., Hasmann, F.A., Jozala, A.F., Penna, T.C., Magalhaes, P.O., Rangel-Yagui, C.O. and Pessoa Jr., A. (2008) Liquid-Liquid Extraction of Biomolecules: An Overview and Update of the Main Techniques. Journal of Chemical Technology \& Biotechnology, 83, 143-157. https://doi.org/10.1002/jctb.1794

[13] Grilo, A.L., Raquel Aires-Barros, M. and Azevedo, A.M. (2016) Partitioning in Aqueous Two-Phase Systems: Fundamentals, Applications and Trends. Separation \& Purification Reviews, 45, 68-80. https://doi.org/10.1080/15422119.2014.983128

[14] Van Berlo, M., Luyben, K.C.A. and van der Wielen, L.A. (1998) Poly(ethylene glycol)-Salt Aqueous Two-Phase Systems with Easily Recyclable Volatile Salts. Journal of Chromatography B: Biomedical Sciences and Applications, 711, 61-68. https://doi.org/10.1016/S0378-4347(97)00627-0

[15] Nalam, P.C., Clasohm, J.N., Mashaghi, A. and Spencer, N.D. (2009) Macrotribological Studies of Poly(L-lysine)-graft-Poly(ethylene glycol) in Aqueous Glycerol Mixtures (PDF). Tribology Letters (Submitted Manuscript), 37, 541-552. https://doi.org/10.1007/s11249-009-9549-9

[16] Hatti-Kaul, R. (2000) Aqueous Two-Phase Systems: Methods and Protocols. Springer Science \& Business Media, Berlin. https://doi.org/10.1385/1592590284

[17] Krasilnikov, O.V., Sabirov, R.Z., Ternovsky, V.I., Merzliak, P.G., Muratkhodjaev, J.N. (1992, September) A Simple Method for the Determination of the Pore Radius of Ion Channels in Planar Lipid Bilayer Membranes. FEMS Microbiology Immunology, 5, 93-100. https://doi.org/10.1111/j.1574-6968.1992.tb05891.x

[18] Bárcena-Uribarri, I., Thein, M., Maier, E., Bonde, M., Bergström, S. and Benz, R. (2013) Use of Nonelectrolytes Reveals the Channel Size and Oligomeric Constitution of the Borrelia burgdorferi P66 Porin. PLoS ONE, 8, e78272. https://doi.org/10.1371/journal.pone.0078272

[19] http://www.ebi.ac.uk/chebi/searchId.do?chebiId=CHEBI:53258.

[20] Masimov, E.A., Shahbazova, G.M., Bagirov, T.O. and Odzhagverdieva, S.Y. (2017) Influence of Some Monohydryc Alcohols on the Phase Diagram "Peg-Sodium Salt of Citric Acid". International Journal of Applied and Fundamental Research, 9, 140-142. https://applied-research.ru/ru/article/view?id=11843 